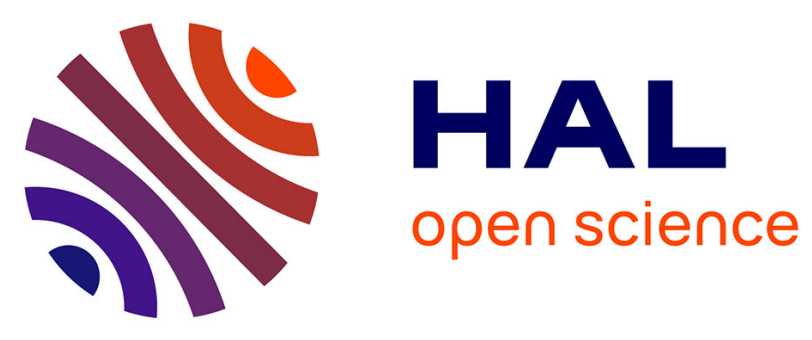

\title{
Amma, une étude multidisciplinaire de la mousson ouest-africaine
}

Jean-Luc Redelsperger, Arona Diedhiou, Cyrille Flamant, Serge Janicot, Jean-Philippe Lafore, Thierry Lebel, Jan Polcher, Bernard Bourlès, Guy Caniaux, Patricia de Rosnay, et al.

\section{To cite this version:}

Jean-Luc Redelsperger, Arona Diedhiou, Cyrille Flamant, Serge Janicot, Jean-Philippe Lafore, et al. Amma, une étude multidisciplinaire de la mousson ouest-africaine. La Météorologie, 2006, 54, pp.22-32. 10.4267/2042/20098 . insu-00385883

\section{HAL Id: insu-00385883 \\ https://hal-insu.archives-ouvertes.fr/insu-00385883}

Submitted on 28 Aug 2009

HAL is a multi-disciplinary open access archive for the deposit and dissemination of scientific research documents, whether they are published or not. The documents may come from teaching and research institutions in France or abroad, or from public or private research centers.
L'archive ouverte pluridisciplinaire HAL, est destinée au dépôt et à la diffusion de documents scientifiques de niveau recherche, publiés ou non, émanant des établissements d'enseignement et de recherche français ou étrangers, des laboratoires publics ou privés. 


\title{
Amma, une étude multidisciplinaire de la mousson ouest-africaine
}

\author{
Jean-Luc Redelsperger (1), A. Diedhiou ${ }^{(2)}$, C. Flamant ${ }^{(3)}$, S. Janicot ${ }^{(4)}$, \\ J.-P. Lafore( ${ }^{(1)}$, T. Lebel ${ }^{(2)}$, J. Polcher ${ }^{(5)}$, B. Bourles ${ }^{(6)}$, G. Caniaux ${ }^{(1)}$, \\ P. De Rosnay ${ }^{(7)}$, M. Desbois ${ }^{(5)}$, L. Eymard( ${ }^{(4)}$, B. Fontaine ${ }^{(8)}$, I. Geneau $^{(9)}$, \\ K. Ginoux ${ }^{(9)}$, M. Hoepffner ${ }^{(10)}$, C. Kane ${ }^{(2)}$, K. Law ${ }^{(3)}$, C. Mari ${ }^{(1)}$, \\ B. Marticorena ${ }^{(13)}$, E. Mougin ${ }^{(7)}$, J. Pelon ${ }^{(3)}$, C. Peugeot $^{(14)}$, A. Protat ${ }^{(12)}$, \\ F. Roux ${ }^{(11)}$, B. Sultan ${ }^{(4)}$, E. Van Den Akker ${ }^{(9)}$ \\ (1) Météo-France - Centre national de recherches météorologiques (CNRM) \\ 42, avenue Gaspard-Coriolis - 31057 Toulouse Cedex \\ redels@meteo.fr \\ (2) Laboratoire d'étude des transferts en hydrologie et environnement (LTHE) - Niamey \\ (3) Service d'aéronomie - Paris \\ (4) Laboratoire d'océanographie et du climat (Locean) - Paris \\ (5) Laboratoire de météorologie dynamique (LMD) - Paris \\ (6) Laboratoire d'études en géophysique et océanographie spatiales (Legos) - Brest \\ (7) Centre d'études spatiales de la biosphère (Cesbio) - Toulouse \\ (8) Centre de recherches de climatologie (CRC) - Dijon \\ (9) Amma Project Office - IPSL - Paris \\ (10) Medias-France - Toulouse \\ (11) Laboratoire d'aérologie - Toulouse \\ (12) Centre d'étude des environnements terrestre et planétaires (CETP) - Vélizy \\ (12) Laboratoire interuniversitaire des systèmes atmosphériques (Lisa) - Créteil \\ (14) Hydrosciences Montpellier (HSM) - Cotonou ${ }^{(1)}$
}

Le projet international Amma, d'initiative française, a pour objectif d'améliorer la connaissance et la compréhension de la mousson d'Afrique de l'Ouest et de sa variabilité, de l'échelle journalière à l'échelle interannuelle. Le projet est motivé par la forte variabilité des précipitations associées à ce système de mousson, et par ses conséquences sur la sécurité alimentaire, les ressources en eau et la santé.

\section{Abstract}

\section{Amma, a multidisciplinary study} of the West African monsoon

Initiated by the French community, the Amma international project aims to improve our knowledge and understanding of the West African monsoon (WAM) and its variability with an emphasis on timescales from daily to interannual. Amma is motivated by an interest in fundamental scientific issues and by the societal need for improved prediction of the WAM and its impacts on water resources, health and food security for West African nations.

e projet Amma (Analyses multidisciplinaires de la mousson africaine) est un projet international dont l'objectif est d'améliorer notre connaissance et notre compréhension de la mousson de l'Afrique de l'Ouest (MAO) ainsi que d'étudier sa variabilité de l'échelle journalière à l'échelle interannuelle et, au-delà, aux échelles décennales. Il a une double ambition, scientifique et sociétale. Les mécanismes fondamentaux d'un système si complexe demandent encore à être mieux compris, dans l'espoir de parvenir à de meilleures prévisions de ses différentes composantes, en particulier la pluviométrie, le cycle hydrologique et les transports de poussière. Ce qui est en jeu, ce sont aussi les conséquences sur la vie des populations locales, leur possibilité de gérer les impacts d'une forte variabilité climatique dans un contexte de pression démographique toujours plus prononcée : dégradation des sols, baisse des rendements agricoles, raréfaction des ressources en eau, résurgence ou renforcement de certaines endémies et épidémies.

Le changement global du climat risque de renforcer l'amplitude des fluctuations de la MAO dans la région et donc d'accroître la vulnérabilité des sociétés africaines. Cette conjonction de facteurs interpelle la communauté scientifique qui, à travers Amma, va impulser la recherche multidisciplinaire nécessaire pour fournir des prévisions améliorées de la $\mathrm{MAO}$, préalable indispensable à l'évaluation de ses impacts. À cette fin, trois grands objectifs ont été fixés.

(1) Les laboratoires et centres cités sont sous diverses tutelles, souvent multiples : plusieurs universités, le CNRS (dont l'Institut PierreSimon-Laplace, IPSL), le Cnes, Météo-France, l'Institut de recherches pour le développement (IRD). 

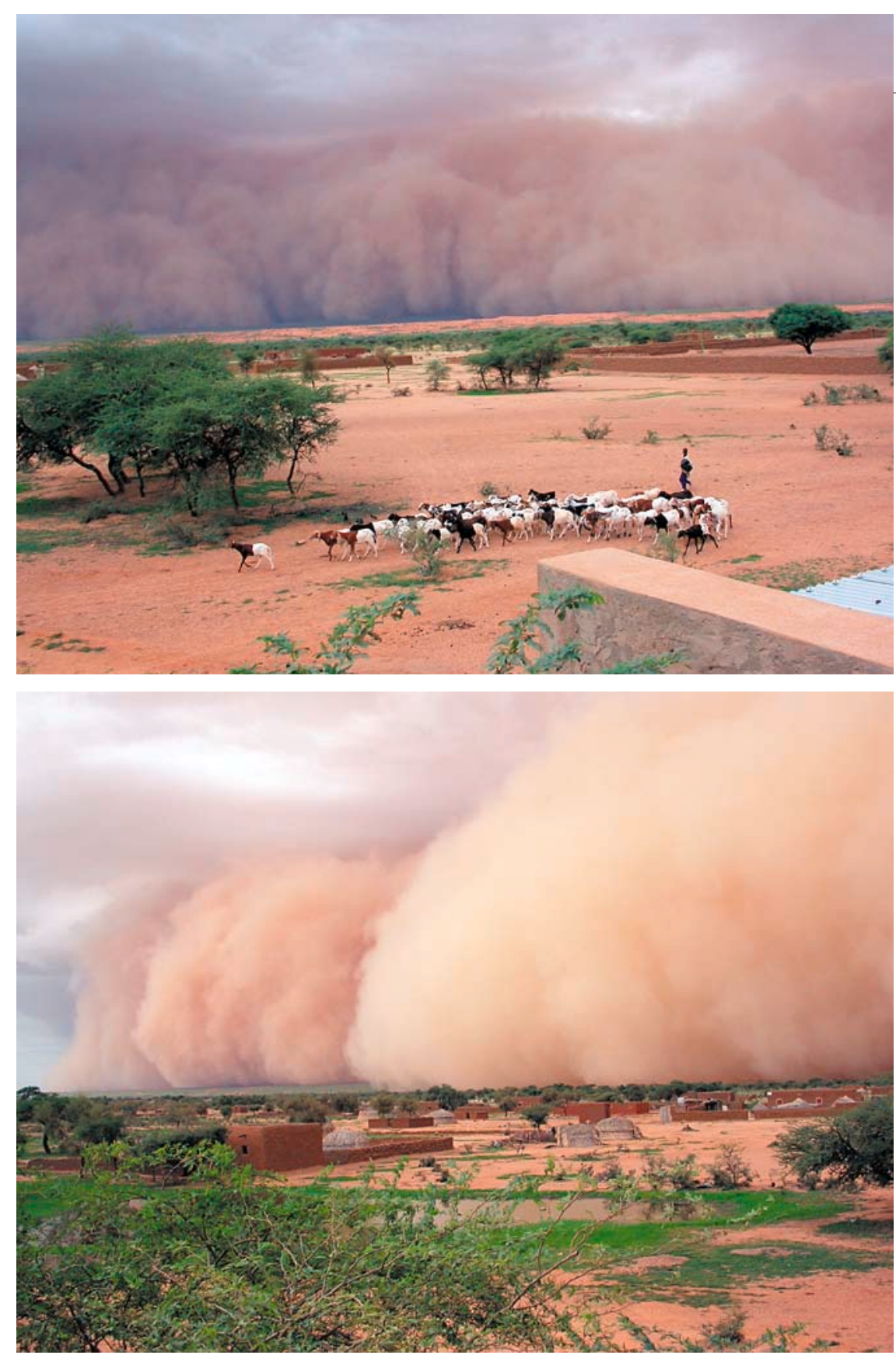

- Améliorer notre compréhension de la mousson africaine, depuis l'échelle des processus élémentaires contrôlant la mousson jusqu'aux échelles auxquelles la MAO influence, au-delà de Afrique

de l'Ouest, la dynamique et la composition de l'atmosphère.

- Produire les connaissances nécessaires pour relier la variabilité du climat aux problèmes de santé, de ressources en eau

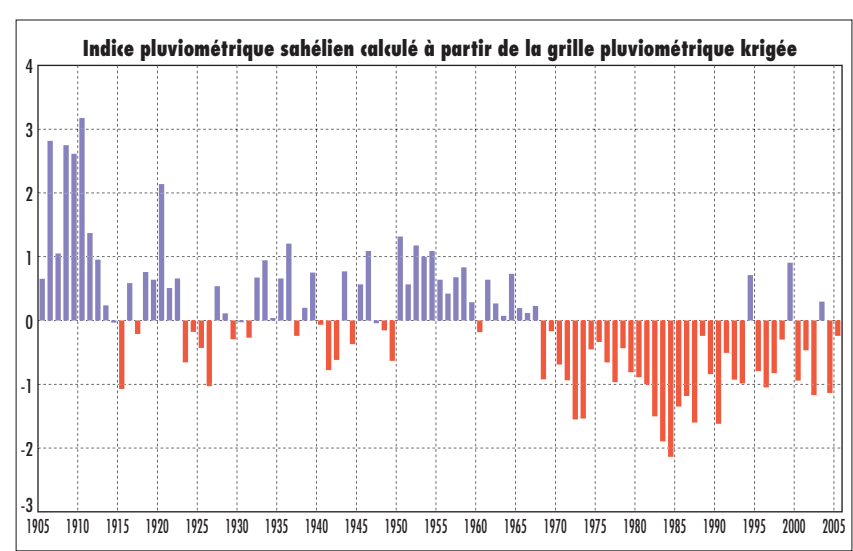

Poussières soulevées à l'avant d'un système convectif, type ligne de grain, au Mali, durant la campagne EOP 2005. (C) CNRS Guichard-Kergoat)

et de sécurité alimentaire de l'Afrique de l'Ouest et définir des stratégies de surveillance appropriées.

- Faire en sorte que les résultats des recherches multidisciplinaires d'Amma soient effectivement intégrés dans les activités de prévision et de décision.

Pour atteindre ces objectifs, Amma s'appuie sur une forte coordination internationale de différentes activités, incluant des recherches fondamentales et des campagnes de mesure menées sur plusieurs années. À travers ces activités, et pour les favoriser, Amma s'attache à resserrer le partenariat entre centres de recherche, centres de prévision opérationnelle et décideurs africains.

\section{Les inconnues sur la mousson africaine}

L'Afrique sub-saharienne dans son ensemble est la région dans le monde qui a connu la plus forte diminution relative des précipitations au cours des cinquante dernières années. La bande sahélienne et soudano-sahélienne est une zone de faibles cumuls annuels de précipitations et de fort gradient méridien de pluviosité, qui a été particulièrement touchée par cette diminution (figures $1 \mathrm{a}$ et $1 \mathrm{~b}$ ). $\mathrm{Au}$ cours du $\mathrm{xx}^{\mathrm{e}}$ siècle, les fluctuations des précipitations sahéliennes ont connu une forte variabilité sur une échelle multidécennale combinant l'occurrence d'une période d'une vingtaine d'années excédentaires suivie d'une trentaine d'années déficitaires. Plus au sud, la région guinéenne a connu une baisse similaire des cumuls annuels de précipitations, se traduisant toutefois par une diminution relative plus faible, la pluie y étant plus élevée qu'au Sahel (Le Barbé et al., 2002).

De nombreuses études ont été menées depuis une trentaine d'années pour comprendre cette évolution. Néanmoins, plusieurs inconnues demeurent, concernant notamment le couplage de la dynamique atmosphérique de la mousson avec les conditions de surface océanique et continentale. Il y a peu encore, la vision qui prévalait était celle d'une dynamique linéaire, la pénétration - puis le retrait du flux de mousson sur le continent étant étroitement associé à la migration du nadir solaire vers le nord, puis à son retrait vers le sud. Des études récentes et la notion de saut de mousson (encadré 


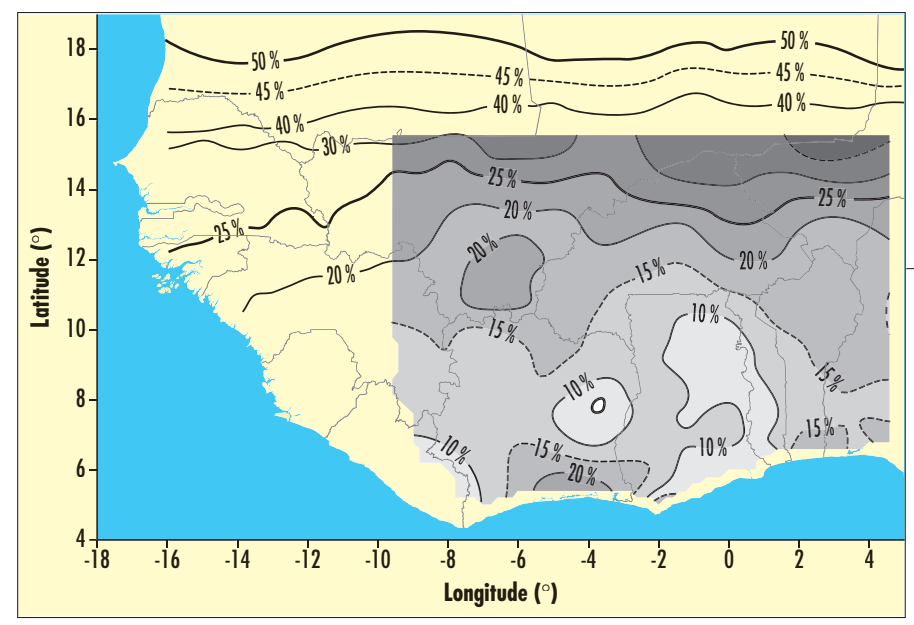

ci-dessous) ont remis en cause cette vision linéaire, sans que l'on comprenne encore bien ce qui pilote le phénomène. D'une façon plus large, on s'interroge toujours sur les causes des modifications de la dynamique de la mousson et des pluies associées, en particulier sur les influences respectives des changements qu'ont connus, aussi bien l'océan (réchauffe-

Figure 1b - Carte du déficit relatif (en pourcentage) de la pluviométrie moyenne annuelle de la période 1970-1989 par comparaison à la période 1950-1969, calculé à partir d'un réseau de 370 stations. Le déficit relatif est particulièrement marqué sur le Sahel : en valeur absolue, il est compris entre 150 et $250 \mathrm{~mm}$ sur la plus grande partie de la région (Ali et al., 2006). La zone grisée est celle où la densité plus importante du réseau a permis une étude comparée du régime pluviométrique de ces deux périodes à l'échelle de l'événement pluvieux (Le Barbé et al., 2002).

ment de $0,5^{\circ} \mathrm{C}$ de l'océan tropical depuis une cinquantaine d'années) que le sous-continent ouest-africain ( profond e dégradation des couverts forestiers). Cette méconnaissance tient en partie à un manque d'observations appropriées, particulièrement sensible du fait que le couplage atmosphère/continent/océan est contrôlé par des interactions d'échelles complexes (ISP, 2005). Or, les réseaux actuellement opérationnels sont hétérogènes et parfois déficients, donc incapables de documenter le spectre d'échelle requis. Certaines variables dont le suivi est nécessaire à la caractérisation du système de mousson, ne sont pas observées en continu (en particulier, il n'existe pas de mesures opérationnelles d'humidité des sols, ni de la couche limite atmosphérique, pas plus que de transport de poussières). La nouvelle génération de satellites va permettre de combler certains de ces manques, mais les données fournies devront faire l'objet de validations détaillées. Quant aux modèles numériques utilisés pour la prévision, ils comportent des biais systématiques sur cette région et ne restituent pas correctement certains traits fondamentaux comme le cycle annuel des précipitations.

\section{Le saut de mousson}

La répartition des précipitations étant quasi zonale sur l'Afrique de l'Ouest, l'utilisation de diagrammes temps-latitude, où les précipitations sont moyennées sur la bande de longitude $10^{\circ} \mathrm{W}-10^{\circ} \mathrm{E}$, permet de résumer efficacement l'information. La figure représente ainsi le cycle annuel moyen des précipitations sur l'Afrique de l'Ouest et le golfe de Guinée. Cette figure montre en fait le déplacement en latitude de ce que l'on appelle la zone de convergence intertropicale (ZCIT) qui est le lieu des plus forts développements convectifs. On voit ainsi sur cette figure plusieurs étapes dans le déplacement en latitude de la ZCIT correspondant aux différentes saisons des pluies présentes en Afrique de l'Ouest.

Entre mi-avril et fin juin, la ZCIT est positionnée au-dessus de la côte guinéenne, ce qui correspond à la première saison des pluies sur cette région. Puis, au cours de la première moitié du mois de juillet, elle se déplace rapidement vers le nord et atteint une latitude relativement stable, autour de $11^{\circ} \mathrm{N}$ en moyenne, correspondant à l'établissement de la saison de mousson d'été sur l'Afrique de l'Ouest, et au plein développement de la saison des pluies sur les régions sahéliennes. Elle reste sur cette latitude jusqu'à la fin du mois d'août, puis redescend progressivement vers le sud, repassant au-dessus de la côte guinéenne pour la deuxième saison des pluies sur cette région.

Cette brusque remontée de la limite méridionale de la ZCIT vers le nord contraste avec son retrait qui apparaît comme une progression plus régulière vers le sud. Elle contraste aussi avec l'évolution de la limite septentrionale (voir par exemple les isolignes 1 à $4 \mathrm{~mm}$ par jour), qui montre une montée progressive vers le nord, moins rapide que la redescente. On observe aussi lors de cette phase de transition rapide une diminution des précipitations et de la convection. C'est ce que l'on a choisi d'appeler le «saut » de mousson, saut apparent dans la mesure où la convection, forte pendant la première saison des pluies sur la côte guinéenne, disparaît temporairement vers la fin du mois de juin pour réapparaître plus au nord peu après le début du mois de juillet. Différentes techniques statistiques ont été appliquées pour identifier ce saut chaque année et déterminer une date qui en repère approximativement le début. Cet exercice n'est pas sans difficulté car, si le saut apparaît très nettement sur une moyenne d'une vingtaine d'années, il n'est pas aussi net systématiquement tous les ans. Cependant, ces méthodes

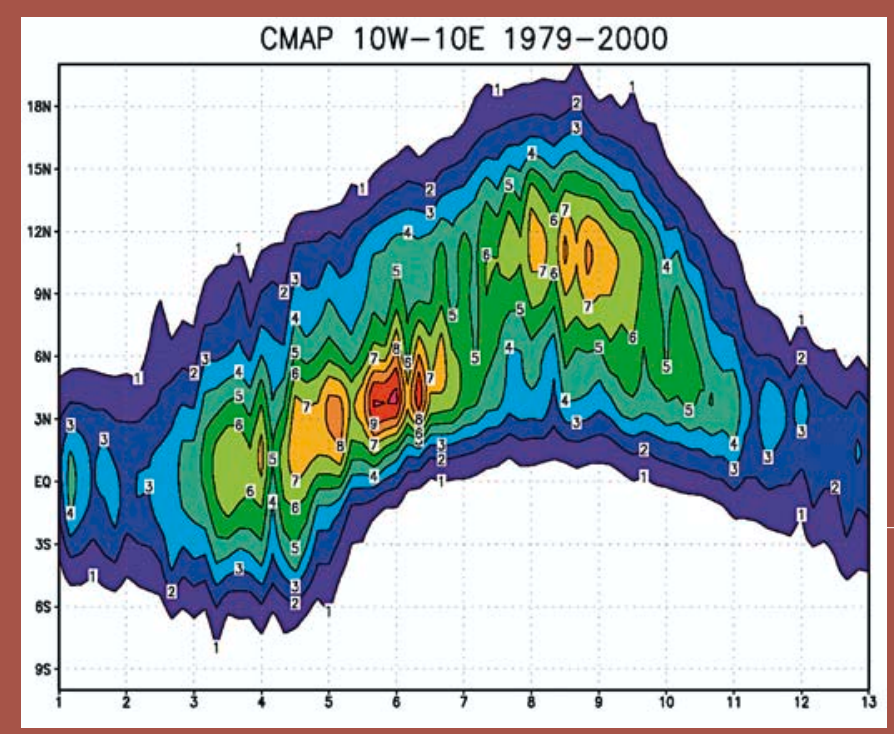
statistiques convergent pour définir une date moyenne autour du 24 juin avec une dispersion moyenne autour de cette date représentée par un écart type de 8 jours environ (Sultan et Janicot, 2003). D'autres études ont montré (par exemple Le Barbé et al., 2002) que ces dates ne sont pas significativement reliées aux cumuls des précipitations durant la mousson d'été. Les mécanismes potentiellement associés à ce saut de la ZCIT sont un des sujets d'étude importants du projet Amma et une des campagnes de terrain de l'été 2006 (la SOP1) est dédiée à cette phase de mise en place de la mousson africaine. L'espoir est de mieux comprendre cette évolution spécifique de la ZCIT que l'on retrouve dans d'autres systèmes de mousson évoluant dans des contextes différents, comme la mousson asiatique.

Diagramme temps-latitude du cycle annuel moyen

des précipitations $(\mathrm{mm})$ moyennées entre $10^{\circ} \mathrm{W}$ et $10^{\circ} \mathrm{E}$ pour la période 1979-2000. Ces données Cmap sont issues de la mise en commun de mesures in situ et d'estimations calculées à partir de mesures satellitaires. La côte guinéenne séparant le golfe de Guinée, au sud, de l'Afrique de l'Ouest, au nord, se situe environ vers $5^{\circ} \mathrm{N}$. (Source S. Louvet) 
En fait, la MAO représente un archétype idéal pour étudier les interactions d'échelles dans un système de mousson (Redelsperger et al., 2002) : elle se caractérise au premier ordre par une symétrie zonale, avec deux jets d'est bien définis en moyenne et haute troposphère ainsi que des entités météorologiques (lignes de grains, ondes d'est) bien identifiées. Étudier de telles interactions d'échelles, et en particulier celles reliant la convection et la dynamique atmosphérique avec les états de surface, sera très utile pour les autres systèmes de mousson et indispensable pour améliorer les modèles couplés océan-continent-atmosphère utilisés pour les prévisions météorologiques et climatiques.

La mousson africaine a des impacts importants sur le climat du reste de la planète. En termes de dynamique atmosphérique, l'énergie de chaleur latente libérée par la convection profonde au-dessus de l'Afrique représente une des sources de chaleur les plus importantes, du point de vue de la circulation atmosphérique planétaire. Il existe aussi un lien étroit entre l'activité de la mousson africaine et la fréquence d'occurrence des cyclones tropicaux sur

\section{Les impacts de la variabilité du climat}

Les populations du Sahel, majoritairement rurales, sont particulièrement concernées par la variabilité climatique dans la mesure où elle conditionne les ressources alimentaires, hydriques et financières, avec des retombées directes sur la santé publique. En effet, les performances de leurs systèmes de production, élevages ou cultures, sont étroitement dépendants du climat (Bazzaz et Sombroek, 1996) ; de plus, celui-ci influe fortement sur la dynamique de maladies meurtrières en Afrique de l'Ouest, comme la méningite, qui affecte entre 25000 et 200000 personnes par an, et le paludisme, responsable de $90 \%$ des décès d'enfants de moins de 5 ans en Afrique.

Cette vulnérabilité aux conditions climatiques ne se retrouve pas dans les pays occidentaux qui ont pu se doter d'infrastructures importantes pour protéger ou atténuer l'impact socio-économique des fluctuations naturelles du climat et des autres ressources naturelles. Mais la plupart de ces infrastructures ont un coût environnemental et financier important ou nécessitent des quantités d'eau et d'énergie difficilement compatibles avec un développement sur le long terme de l'Afrique.

L'évolution climatique récente est un exemple frappant de ces impacts sur les populations. Ainsi, les sécheresses des années 1970 ont provoqué une famine dramatique et celles des années 1980 ont décimé les troupeaux (PNUD, 2004). C'est dans ce contexte d'impacts socio-économiques forts, auxquels s'ajoute la perspective de changement global lié à l'augmentation des gaz à effet de serre, qu'il est apparu essentiel à la communauté scientifique de décrire et de comprendre les modes de variabilité du climat en Afrique de l'Ouest pour en améliorer les prévisions. On ne doit pas s'arrêter là, il faut aussi développer les méthodes et outils ainsi que l'expertise en Afrique qui permettra d'appliquer ces prévisions aux activités humaines. II sera alors possible d'apporter une aide aux décideurs pour choisir une stratégie agricole ou planifier une campagne ciblée de vaccination.

Les applications et études d'impacts (ressources en eau, agronomie, santé publique, socio-économie) sont ainsi l'un des enjeux ultimes d'Amma. Climatologues, hydrologues, agronomes, biologistes, médecins, mais aussi acteurs locaux, sont réunis au sein de ce projet pour répondre à des objectifs communs. Une première série d'objectifs est liée à une meilleure représentation du cycle de l'eau de l'échelle locale à l'échelle régionale. Il s'agit notamment :

- d'établir l'importance respective des fluctuations climatiques et des modifications environnementales sur les ressources en eau afin de proposer des stratégies de dimensionnement d'ouvrages et de gestion des ressources prenant en compte les prévisions climatiques

- de mesurer et de prévoir les impacts de la variabilité du climat à grande échelle sur les échelles fines de la parcelle agricole pour permettre aux techniciens du monde rural de mieux orienter les calendriers agricoles et d'optimiser les recherches agronomiques en matière de sélection variétale et d'adaptations de pratiques agricoles;

- de mieux cerner les facteurs de prévalence du paludisme qui est fortement lié à I'humidité et aux précipitations en été (Craig et al., 1999).

Une meilleure connaissance des liens entre la dynamique atmosphé-

rique, les conditions de surface et les transports d'aérosols doit par ailleurs permettre de déterminer des types de temps susceptibles de favoriser la survenue des épidémies de méningites (figure ci-contre, vents secs d'harmattan en hiver, Sultan et al., 2005) ou la dégradation des sols.

Relations entre la date du démarrage de l'épidémie de méningite au Mali et la date de maximum de vents d'harmattan en hiver. Les dates sont exprimées en semaine calendaire. (Sultan et al., 2005)

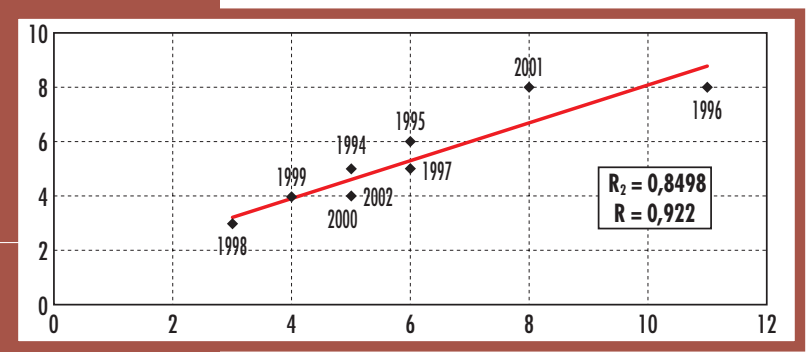

l'Atlantique. Le lien entre mousson africaine et climat planétaire vaut aussi pour la chimie atmosphérique. L'Afrique représente une source importante d'émissions naturelles et anthropogéniques de composés gazeux et particulaires. Ainsi, $20 \%$ des émissions par les feux de biomasse trouvent leur source en Afrique de l'Ouest. Les précurseurs gazeux émis sur le continent ont un impact important sur la concentration en ozone troposphérique (gaz à effet de serre) dans la zone tropicale. Plus généralement, le transport à longue distance de ces gaz traces participe à la modification de la capacité oxydante de l'atmosphère, qui contrôle le niveau de concentration de nombreux gaz à effet de serre, et contribue au transfert de constituants clés dans la stratosphère (vapeur d'eau et éléments influant sur la concentration d'ozone). L'Afrique est également la source principale d'aérosols atmosphériques dans le monde. Ainsi, la moitié des émissions globales d'aérosols désertiques provient de l'ensemble Sahara-Sahel. Qu'elles soient produites par l'érosion éolienne ou les feux de biomasse, ces particules ont un impact important sur le forçage radiatif, du domaine UV à l'infrarouge, et sur la microphysique des nuages. Elles constituent ainsi une composante importante du système de mousson africain. Dans ce contexte, il est donc nécessaire d'étudier simultanément la dynamique et ses interactions avec la composition gazeuse et particulaire de l'atmosphère.

La documentation géophysique de la $\mathrm{MAO}$ à différentes échelles de temps et d'espace est donc au cœur des efforts à mener à court terme. C'est le préalable indispensable pour mener les études d'impacts et les applications socioéconomiques initiées dans le cadre d'Amma et qui se développeront sur le long terme. Ce programme devrait ainsi permettre une meilleure intégration des recherches fondamentales sur le fonctionnement de la mousson, menées par les géophysiciens, et des recherches qui s'intéressent au milieu humain et à son environnement, menées par les géographes, les agronomes et les socioéconomistes (encadré ci-contre). 


\section{Une approche intégrée à travers les échelles et les disciplines}

Dans le domaine géophysique, les enjeux scientifiques majeurs du projet Amma s'organisent autour de trois sujets fédérateurs :

- les interactions de la MAO et du climat global du point de vue de la dynamique et de la chimie atmosphériques ; - le cycle de l'eau associé à la MAO ;

- le couplage atmosphère, surfaces continentales et océaniques.

Ces deux derniers sujets sont par essence multiéchelles. Pour nourrir ces questions, des recherches spécifiques sur les processus sont nécessaires dans quatre grands domaines :

- l'atmosphère et ses processus nuageux convectifs et précipitants ;

- les processus d'échanges océaniques ; - les processus physiques et biophysiques sur les surfaces continentales ;

- les aérosols et la chimie atmosphérique.

En ce qui concerne les dimensions environnementales et socio-économiques, l'identification et la quantification de l'impact des fluctuations interannuelles et à long terme de la $\mathrm{MAO}$ requièrent d'accorder une importance particulière aux questions d'échelle. En effet, alors que la MAO est un système climatique régional dont la prévision s'effectue à l'aide de modèles de grande échelle, les effets de sa variabilité se font sentir localement de manière très contrastée, en fonction des facteurs environnementaux et humains. L'intégration disciplinaire recherchée dans Amma passe donc par une grande attention portée à la définition des échelles d'études pertinentes et aux problèmes métrologiques et méthodologiques qui apparaissent dès que l'on cherche à mettre en relation des effets locaux avec la variabilité d'un système régional.

Pour tenir compte de ces problématiques foncièrement multiéchelles, le programme Amma est structuré autour de quatre échelles spatiales (figure 2).

\section{L'échelle planétaire}

C'est l'échelle à laquelle la MAO interagit avec le reste du globe. À cette échelle, Amma se focalise sur le rôle des anomalies globales de températures de surface de la mer sur la variabilité de la $\mathrm{MAO}$, ainsi que sur l'impact de cette

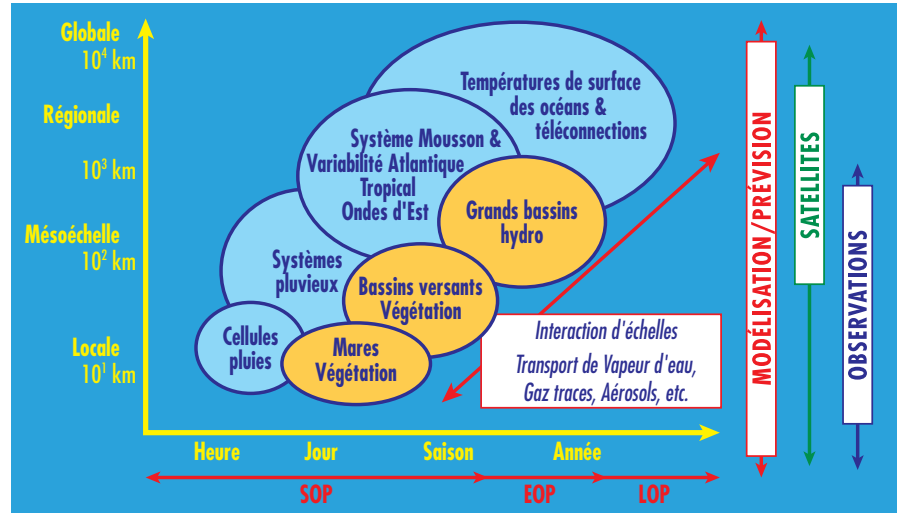

Figure 2 -

Les échelles d'intérêts de la mousson africaine en regard de ses processus clés et de ses composantes (Amma International Science Plan 2005).

variabilité sur les processus dynamiques et 1 'exportation à grande distance de composés atmosphériques (particules et gaz) au-dessus de l'Atlantique tropical. On étudie également les différents facteurs qui conditionnent la dynamique de la mousson et l'abondance des pluies associées, dans le but de cerner l'origine de leurs variabilités, des saisonnières aux échelles décennales.

\section{L'échelle régionale}

Les processus régissant les interactions entre échelles internes à la mousson doivent être étudiés à l'échelle régionale. L'accent est mis sur la compréhension des interactions entre l'atmosphère, la surface continentale et l'océan (spécialement dans le golfe de Guinée). On étudiera, par exemple, le rôle des rétroactions des surfaces continentales sur la variabilité de la MAO. C'est aussi l'échelle caractéristique des processus de transport des composés atmosphériques par la MAO. Cette échelle spatiale permet une étude plus fine des cycles moyens annuels et saisonniers, ainsi que de leurs fluctuations interannuelles.

\section{La mésoéchelle}

C'est celle des systèmes convectifs précipitants, composante météorologique majeure de la MAO. Cette échelle est centrale pour l'étude de la variabilité saisonnière des précipitations et le couplage entre hydrologie et atmosphère au niveau des bassins versants. Il en est de même pour traiter du rôle des systèmes convectifs dans le transport à haute altitude des aérosols et des composés chimiques.

\section{Échelle subméso (inférieure à $10 \mathrm{~km}$ )}

D'un point de vue atmosphérique, c'est l'échelle des cellules convectives auxquelles correspondent les maxima de précipitations. À ce titre, c'est l'échelle d'étude pour les processus microphysiques et dynamiques conduisant à la formation de précipitations ; pour l'hydrologie des petits bassins versants, qui est déterminante à la fois pour les ressources en eau et pour la propagation des maladies liées au cycle de l'eau (notamment le paludisme) ; pour les processus liés à la végétation naturelle et cultivée.

Une partie de l'équipe Amma à pied d'œuvre devant l'ATR-42 de Safire. (Photo IRD, T. Lebel)

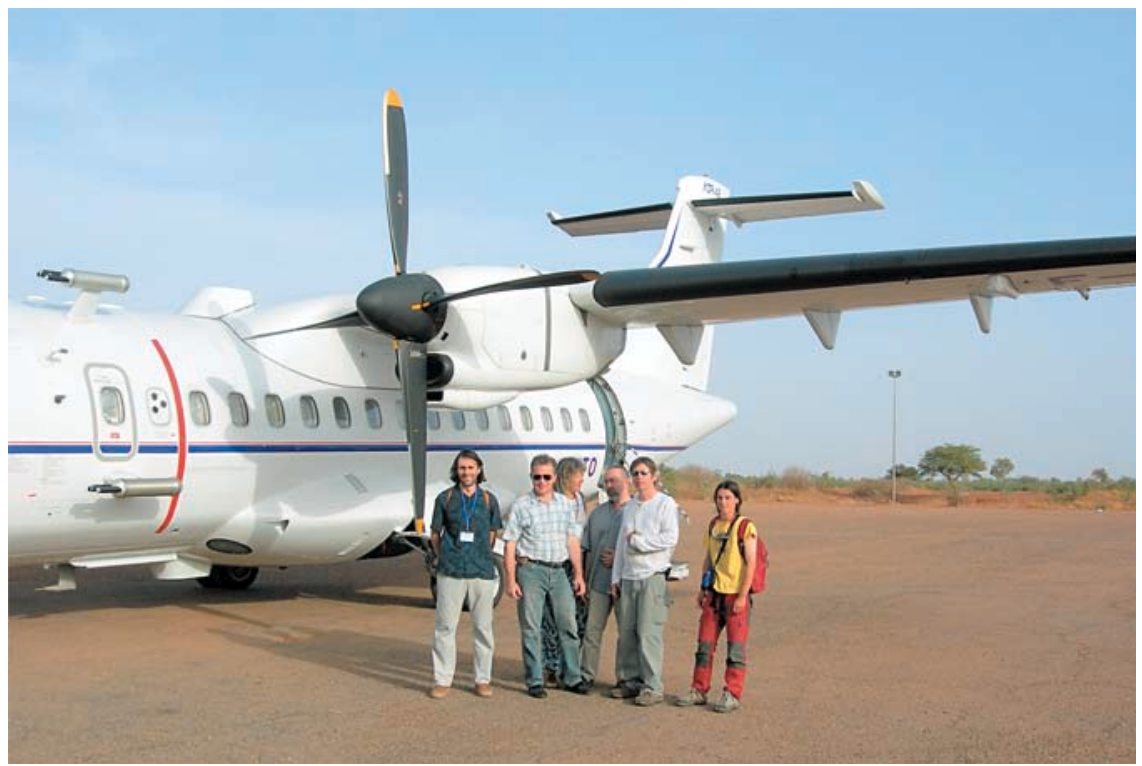




\section{Les expériences de terrain}

Le caractère multiéchelle du système étudié a conduit à définir une stratégie de campagne reposant sur l'imbrication des domaines et des périodes d'observation, et sur l'utilisation de différentes plateformes instrumentées complémentaires (bateaux, sites au sol, ballons, moyens aéroportés, capteurs spatiaux). Cette stratégie résulte également des compromis à trouver en regard du coût de déploiement d'instruments lourds et de leurs limitations en termes d'échantillonnage temporel et spatial, alors même que l'on cherche à documenter l'ensemble du spectre des échelles mises en jeu.

Le premier objectif est d'accumuler des données sur le gradient écoclimatique qui caractérise avant tout la région d'expérience. Le second est de couvrir les différentes échelles identifiées plus haut en combinant des capteurs aux propriétés complémentaires. Par exemple, les moyens aéroportés fournissent des cas d'étude très finement documentés sur des zones assez vastes, tandis que les observations au sol ne permettent qu'un suivi local mais continu temporellement. Pour optimiser l'échantillonnage, les mesures au sol sont organisées en réseaux à couverture régionale relativement homogène et en sites densément instrumentés (figure 3 ).

\section{Un emboîtement de zones de mesure}

- À l'échelle régionale, une priorité incontournable est le renforcement des capacités des réseaux opérationnels sur le continent et plus spécifiquement du réseau de radiosondages (une vingtaine de stations de différents niveaux de priorité selon la période de mesure considérée). Pour cette échelle, sont donc pertinentes les mesures océaniques réalisées au cours des campagnes Egee, la mise en place d'un transect sahélien pour le suivi journalier des aérosols désertiques, et les données pour plusieurs bassins versants et sites de mésoéchelle assurant un bon échantillonnage de toute la région.

- Les études de mésoéchelle sont concentrées sur trois sites d'observations densifiées du cycle hydrologique, de la végétation et des flux de surface : Gourma malien, Kori de Dantiandou dans le Sud-Ouest nigérien, haut bassin de l'Ouémé au Bénin. (Figure 3).

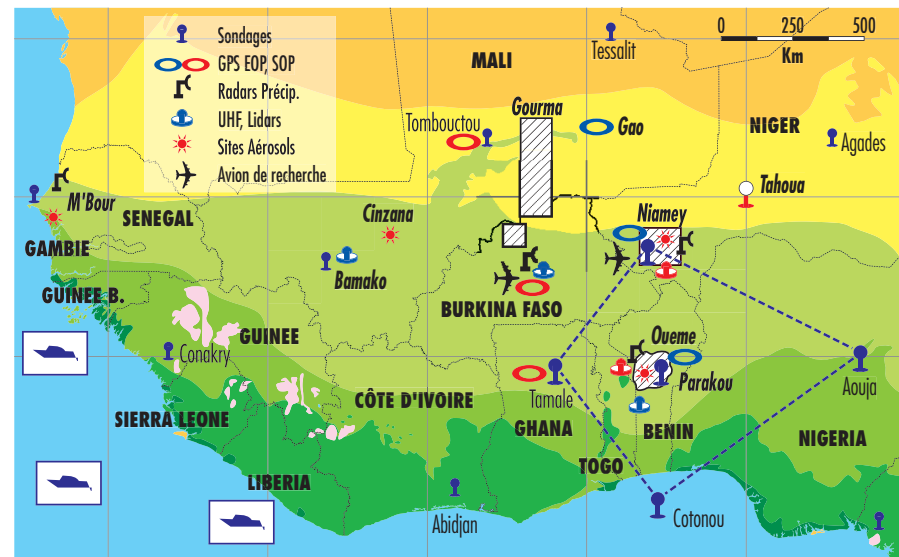

Figure 3 -

Le dispositif régional pour les campagnes. Les sites de mésoéchelle sont les zones hachurées.

- L'échelle convective/locale est l'échelle des supersites $\left(100\right.$ à $\left.1000 \mathrm{~km}^{2}\right)$ ou de sites intensifs locaux localisés à l'intérieur des sites de mésoéchelle.

\section{Un emboîtement de périodes de mesure}

Ces échelles spatiales conditionnent en grande partie les échelles temporelles d'observation. Ainsi, les différentes composantes du cycle de l'eau à l'origine de la variabilité interannuelle de la mousson africaine et l'influence de cette variabilité sur la composition atmosphérique font l'objet d'un Programme d'observations à long terme (LOP), s'étendant de 2001 à 2010. L'objectif est de documenter finement le cycle saisonnier moyen et ses modulations entre années sèches et humides. Ce suivi décennal permettra également d'étudier les effets cumulatifs des séries d'années sèches et humides. Le LOP s'appuie essentiellement sur trois Services d'observations (SO) labellisés par l'Insu sur l'Afrique de l'Ouest : Amma-Catch est dédié à l'étude du cycle hydrologique et de son couplage avec la dynamique de la végétation; Idaf et Photons permettent l'enregistrement à long terme des contenus en aérosols, des concentrations en gaz traces et de la composition des dépôts humides. En complément, l'évolution de la couverture végétale, qui contrôle le bilan hydrique et les émissions d'aérosols terrigènes ou carbonés, sont suivies sur le terrain et par télédétection.

Durant une période d'observations renforcées (EOP) de trois ans (20052007), le dispositif LOP est renforcé pour fournir des observations plus détaillées sur certains processus couplés, susceptibles de jouer un rôle important dans la variabilité interannuelle. Les compartiments concernés couche limite de surface et couche limite atmosphérique, profils océagrées et distribuées sur la verticale sont particulièrement difficiles à instrumenter. Ce dispositif regroupe 41 instruments $^{(1)}$, souvent sophistiqués et coûteux à déployer, ce qui explique la durée limitée de cette période d'observation renforcée.

Le cœur du dispositif expérimental Amma est constitué par la période d'observations spéciales (SOP, 2006). Elle a pour objectif principal l'acquisition de jeux de données permettant d'étudier de façon détaillée une saison de mousson en termes de processus, et plus particulièrement les systèmes météorologiques lors des différentes phases de la mousson :

- la saison sèche précédant la mousson (janvier - février) ;

- l'établissement de la mousson (juin); - la mousson au stade mature (juilletaoût) ;

- la fin de la mousson (septembre).

$\mathrm{Au}$ sein de ces différentes phases, des moyens aéroportés et au sol seront déployés de façon coordonnée et selon une stratégie d'intégration des échelles et des disciplines incluant les données spatiales et la modélisation numérique.

\section{6 : I'année des observations intensives}

La saison sèche est caractérisée par la présence de fortes concentrations d'aérosols désertiques ou issus de la combustion de la biomasse. L'objectif de la SOP 0

(1) Un instrument est défini comme un ensemble de capteurs, ou un capteur unique, fournissant un jeu de données homogènes - en termes de variable mesurée, de résolution, de fréquence d'échantillonnage et de zone couverte : par exemple, un ensemble de stations de radiosondages couvrant une sous-région particulière, un réseau de pluviographes sur un site de mésoéchelle, un radar. Les 41 instruments EOP représentent ainsi plusieurs centaines de capteurs répartis sur toute la région d'étude. 


\section{Sigles utilisés}

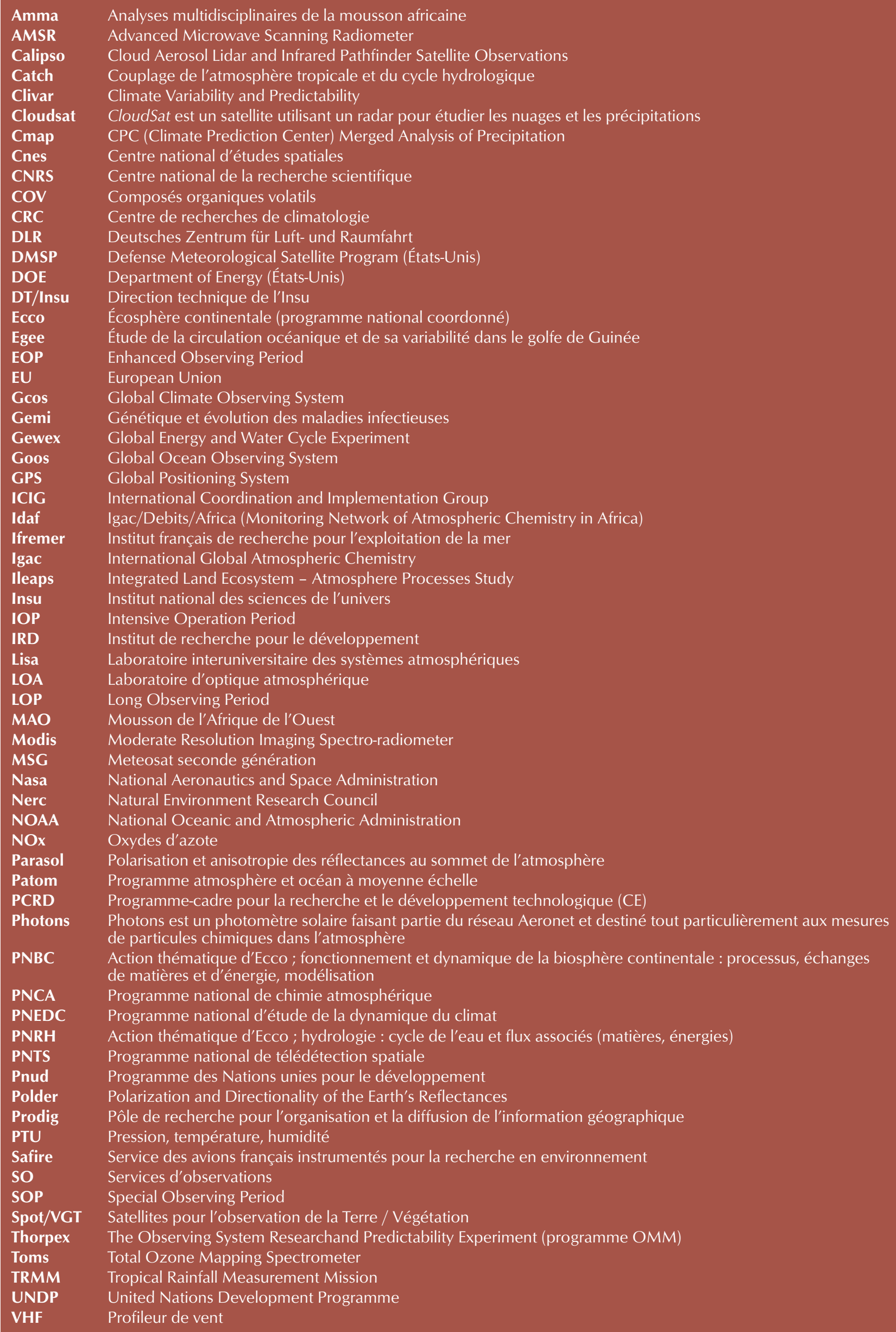

Amma

AMSR

Calipso

Catch

Clivar

Cloudsat

Cmap

Cnes

CNRS

COV

CRC

DLR

DMSP

DOE

DT/Insu

Ecco

Egee

EOP

EU

Gcos

Gemi

Gewex

Goos

GPS

ICIG

Idaf

Ifremer

Igac

lleaps

Insu

IOP

IRD

Lisa

LOA

LOP

MAO

Modis

MSG

Nasa

Nerc

NOAA

NOx

Parasol

Patom

PCRD

Photons

PNBC

PNCA

PNEDC

PNRH

PNTS

Pnud

Polder

Prodig

PTU

Safire

SO

SOP

Spot/VGT

Thorpex

Toms

TRMM

UNDP

VHF

Analyses multidisciplinaires de la mousson africaine

Advanced Microwave Scanning Radiometer

Cloud Aerosol Lidar and Infrared Pathfinder Satellite Observations

Couplage de l'atmosphère tropicale et du cycle hydrologique

Climate Variability and Predictability

CloudSat est un satellite utilisant un radar pour étudier les nuages et les précipitations

CPC (Climate Prediction Center) Merged Analysis of Precipitation

Centre national d'études spatiales

Centre national de la recherche scientifique

Composés organiques volatils

Centre de recherches de climatologie

Deutsches Zentrum für Luft- und Raumfahrt

Defense Meteorological Satellite Program (États-Unis)

Department of Energy (États-Unis)

Direction technique de l'Insu

Écosphère continentale (programme national coordonné)

Étude de la circulation océanique et de sa variabilité dans le golfe de Guinée

Enhanced Observing Period

European Union

Global Climate Observing System

Génétique et évolution des maladies infectieuses

Global Energy and Water Cycle Experiment

Global Ocean Observing System

Global Positioning System

International Coordination and Implementation Group

Igac/Debits/Africa (Monitoring Network of Atmospheric Chemistry in Africa)

Institut français de recherche pour l'exploitation de la mer

International Global Atmospheric Chemistry

Integrated Land Ecosystem - Atmosphere Processes Study

Institut national des sciences de l'univers

Intensive Operation Period

Institut de recherche pour le développement

Laboratoire interuniversitaire des systèmes atmosphériques

Laboratoire d'optique atmosphérique

Long Observing Period

Mousson de l'Afrique de l'Ouest

Moderate Resolution Imaging Spectro-radiometer

Meteosat seconde génération

National Aeronautics and Space Administration

Natural Environment Research Council

National Oceanic and Atmospheric Administration

Oxydes d'azote

Polarisation et anisotropie des réflectances au sommet de l'atmosphère

Programme atmosphère et océan à moyenne échelle

Programme-cadre pour la recherche et le développement technologique (CE)

Photons est un photomètre solaire faisant partie du réseau Aeronet et destiné tout particulièrement aux mesures de particules chimiques dans l'atmosphère

Action thématique d'Ecco; fonctionnement et dynamique de la biosphère continentale : processus, échanges de matières et d'énergie, modélisation

Programme national de chimie atmosphérique

Programme national d'étude de la dynamique du climat

Action thématique d'Ecco; hydrologie : cycle de l'eau et flux associés (matières, énergies)

Programme national de télédétection spatiale

Programme des Nations unies pour le développement

Polarization and Directionality of the Earth's Reflectances

Pôle de recherche pour l'organisation et la diffusion de l'information géographique

Pression, température, humidité

Service des avions français instrumentés pour la recherche en environnement

Services d'observations

Special Observing Period

Satellites pour l'observation de la Terre / Végétation

The Observing System Researchand Predictability Experiment (programme OMM)

Total Ozone Mapping Spectrometer

Tropical Rainfall Measurement Mission

United Nations Development Programme

Profileur de vent 
Le Falcon 20 de l'unité Safire. (Photo Météo-France, J.-M. Destruel)

est donc de documenter précisément les propriétés optiques et physico-chimiques de ces deux types d'aérosols, notamment pour des situations de mélange. Elle s'appuie sur trois sites instrumentés au sol (M'Bour, Sénégal ; BanizoumbouNiamey, Niger ; Djougou, Bénin) et sur des mesures aéroportées du BAe 146 (Royaume-Uni). L'ensemble de ces mesures sera utilisé afin d'estimer le forçage radiatif lié à l'effet direct des aérosols, de l'échelle locale à l'échelle régionale. Ce travail permettra de mieux représenter la distribution des aérosols et leur impact radiatif dans les modèles atmosphériques, d'échelle régionale à globale. Ces données permettront également d'améliorer ou de valider les produits aérosols par satellites.

L'objectif général des SOP 1 et 2 est d'améliorer la connaissance des interactions entre les processus impliqués dans le système MAO : dynamique, microphysique et chimie (incluant les aérosols) de l'atmosphère, surfaces continentales et océan. La stratégie d'observation est telle qu'elle doit permettre de suivre ces processus tout au long de la saison de mousson, grâce aux observations sol, mais aussi d'effectuer, avec les ballons et les avions, des mesures ponctuelles mais plus détaillées, et en particulier d'encadrer le saut de mousson pour analyser les interactions à l'œuvre. Au cours de la phase mature de la mousson, ce sont différentes rétroactions sur le système MAO qui seront étudiées en détail : composition chimique de la troposphère et son interaction avec les systèmes convectifs, propriétés microphysiques, dynamiques et radiatives des enclumes de systèmes convectifs, rôle de réservoir thermique océanique, des intrusions d'air sec, etc.

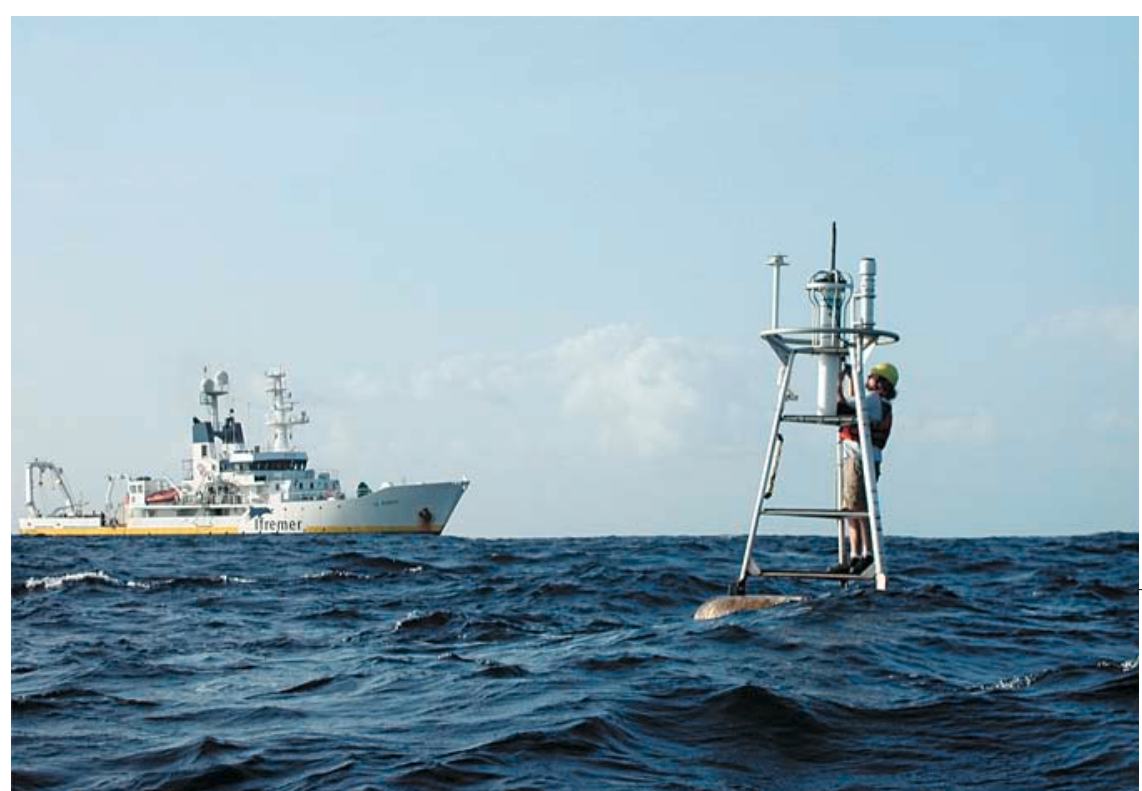

posphère et stratosphère. Trois bateaux seront également déployés dans l'Atlantique tropical et le golfe de Guinée. Les mesures au sol sur les supersites seront encore intensifiées, avec en particulier le déploiement de radars, lidars et radiomètres au cours de la SOP. Les cadences de radiosondage (PTU, ozone) seront également augmentées à cette période.

Au cours de la SOP 3, l'objectif généra est de mieux comprendre le devenir des systèmes convectifs africains au-dessus de l'océan, et notamment le rôle des interactions entre l'océan, les ondes d'est et la couche d'air saharien sur l'évolution de ces systèmes en tempêtes tropicales, puis en cyclones tropicaux.

La SOP verra le déploiement de six avions de recherche : le BAe 146, le Falcon 20 et 1'ATR-42 de Safire (Service des avions français instrumentés pour la recherche en environnement, France), le Falcon 20 du DLR (Allemagne), le M 55 du consortium européen Geophysica et le DC 8 de la Nasa. Les configurations instrumentales de ces avions évolueront selon les différentes périodes d'observations intensives (IOP), en fonction des objectifs scientifiques. Parallèlement, plusieurs campagnes ballons sont programmées en couche limite, haute tro-

\section{La composante satellite d'Amma}

Les données de satellites représentent le seul moyen d'accéder à des observations de l'ensemble du continent ouestafricain, et cela à des échelles de temps comprises entre un jour et plusieurs années. Elles permettent de spatialiser les mesures de terrain à des échelles supérieures et ainsi d'évaluer et d'améliorer les modèles numériques utilisés dans Amma. Tous les domaines thématiques sont couverts et la structuration suit une logique d'échelle (plutôt que disciplinaire) évitant un cloisonnement thématique. Les domaines de restitution des paramètres correspondent à une hiérarchie d'échelles avec trois domaines emboîtés : globe à résolution lâche pour les études climatiques (par exemple température des océans), région de l'Afrique de l'Ouest où les meilleures résolutions seront conservées selon les possibilités d'archivage et trois domaines locaux correspondant aux sites d'instrumentations au sol renforcées. La résolution temporelle des différents produits est choisie en premier lieu en fonction de la résolution des capteurs utilisés, puis suivant l'échelle considérée et la variabilité temporelle du paramètre géophysique.

L'Atalante, le navire de mesures de I'lfremer avec une bouée Pirata. (@ IRD, Bernard Bourles) 
Les produits existants, s'il s'avèrent de qualité satisfaisante et s'ils sont adaptés aux objectifs d'Amma, sont collectés et centralisés. Dans le cas contraire, des produits améliorés sont mis au point et validés. Cela concerne en particulier les précipitations, un facteur clé de l'analyse de la MAO. Un effort a donc été entrepris dans ce domaine, en utilisant les satellites $M S G$, TRMM, DMSP et des réseaux de validation au sol mis spécialement en œuvre à l'occasion d'Amma.

Pour l'atmosphère et l'océan, de nombreux produits sont déjà fournis par divers centres ou services (classifications nuageuses, température et humidité, vents déduits des déplacements nuageux, précipitations, températures et vents à la surface de la mer, flux de surface, flux radiatifs au sommet de l'atmosphère, aérosols, chimie atmosphérique...).

Amma offre par ailleurs l'occasion de valider les produits de satellites par des mesures de terrain. Ce sera le cas notamment des produits dérivés des mesures effectuées par la mission Nasa/Cnes «A-Train », constituée de six satellites en formation, dont Parasol (instrument Polder), Calipso (lidar et imageur infrarouge) et Cloudsat (radar nuage), qui contribueront à l'étude fine des nuages et des aérosols. Un effort de validation des produits disponibles pour l'étude des surfaces continentales (végétation ; occupation des sols; humidité...) sera mené à partir des mesures réalisées sur les sites de mésoéchelle, notamment pour les produits Spot/VGT, Modis et AMSR.

\section{Exemple du site mésoéchelle de l'Ouémé}

La haute vallée de l'Ouémé est l'un des trois sites de mésoéchelle instrumentés de manière intensive dans le cadre de Amma. Le bassin couvre $14600 \mathrm{~km}^{2}$ en climat soudanien, avec une pluie moyenne interannuelle allant de $1100 \mathrm{~mm}$ à $1450 \mathrm{~mm}$ (moyenne sur la période : $1250 \mathrm{~mm}$ ). La végétation est constituée d'une savane arborée, avec de larges espaces cultivés en coton et cultures vivrières.

\section{Le LOP}

Lors de la sécheresse des années 1970 et 1980 , la pluviométrie a baissé de $18 \%$ mais le débit de l'Ouémé à Bétérou a été divisé par deux (figure 4). La pression démographique est forte $(4,9 \%$ de croissance annuelle), du fait notamment d'une immigration en provenance du nord-ouest du pays, ce qui entraîne une évolution rapide du couvert végétal. Le suivi hydrologique de plusieurs bassins emboîtés (figure 5), mis en place à la fin des années 1990, a pour but étudier les interactions entre dynamique de ce couvert végétal en évolution et cycle de l'eau.

\section{La zone EOP}

Sur cette région, il existe un décalage de plusieurs semaines entre les premières pluies significatives d'avril et la mise en eau du réseau hydrographique. Cela représente en moyenne une lame d'eau de $250 \mathrm{~mm}$, stockée dans la zone d'altérites accessibles à la végétation ou bien dans le réservoir d'eau souterraine. Le dispositif du LOP a été renforcé en 2005 pour mieux cerner les mécanismes qui pilotent ces phénomènes de stockage et déstockage : stations de mesures de flux et scintillomètre, capteurs de suivi de l'humidité des sols, campagnes géophysiques et géochimiques pour identifier les temps de résidence de l'eau dans les différents compartiments. Un radar polarisé en bande $\mathrm{X}$ sert à documenter finement la variabilité des précipitations pour aider au calcul de bilans d'eau précis sur les différents bassins. Ces calculs viennent en appui à la modélisation hydrologique (validation) et aux études de ressources en eau ; ils fourniront également la composante continentale des bilans d'eau couplés atmosphère-surface dont l'évaluation est l'un des objectifs majeurs de la SOP sur cette région.

Un dispositif de mesures chimiques installé à Nangatchori permettra de bâtir un inventaire des émissions de sources biogéniques (NOx, COV) ou liées à la
Figure 4 - Variabilité interannuelle des pluies et des écoulements sur le bassin du haut Ouémé (9 670 km²). La sécheresse des années 1970 et 1980 est bien visible. L'un des objectifs du suivi LOP-EOP est de comprendre comment la variabilité interannuelle des bilans d'eau est reliée à la variabilité de la mousson elle-même et à la dynamique des couverts végétaux.

combustion de biomasse (savane et feux domestiques). Les données EOP ainsi obtenues serviront à l'assimilation dans les modèles régionaux et à leur validation. Comme sur les deux autres sites de mésoéchelle, un photomètre et un lidar donnent des mesures d'épaisseur optique et d'absorption, couplées à des mesures de bilan radiatif.

\section{La SOP}

Un des axes de la SOP est ici l'observation des systèmes convectifs, qui présentent une diversité plus grande qu'au Sahel et une forte modulation saisonnière (avant / après le saut de mousson). La figure $5 \mathrm{c}$ montre le dispositif réuni pour cela : il vise à la fois à une documentation fine de la colonne atmosphérique (site intensif de Nangatchori) et de la dynamique spatiale des systèmes convectifs (radars et réseau de détecteurs d'éclairs).

Le bassin de l'Ouémé est aussi au centre du quadrilatère d'étude des bilans d'eau atmosphériques, défini par les stations de radiosondage de Cotonou (Sud), Tamale (Est), Niamey (Nord) et Abuja (Ouest), avec la station de Parakou en son centre. Sur cette zone, on pourra comparer les bilans calculés par les modèles hydrologiques avec ceux calculés par les modèles atmosphériques. Les mesures verticales EOP/SOP (VHF, GPS, radiosondages) vont permettre d'obtenir avec une résolution inhabituelle les fluctuations du flux du mousson et les transports associés.

\section{Modélisations du temps et du climat}

Les modèles représentent la synthèse de nos connaissances sur la physique et la chimie de l'atmosphère, l'océan et les surfaces continentales, mais aussi les outils qui offrent les meilleures promesses pour prévoir l'évolution de l'environnement à toutes échelles de temps et d'espace. La nature parcellaire de nos connaissances de la MAO est reflétée 
Figure 5 - Les différents dispositifs de mesure sur le bassin de l'Ouémé, un des trois sites de mésoéchelle Amma. (a), dispositif EOP sur l'ensemble du bassin ; (b), dispositif EOP sur le supersite de la Donga; (c) instruments SOP, essentiellement concentrés sur la Donga, avec un réseau de détecteurs d'éclairs (anneaux verts) couvrant tout le bassin de l'Ouémé.

par la variété des processus représentés dans nos différents modèles allant des processus de petite échelle (par exemple, les bassins endoréiques du Sahel) jusqu'au transport à l'échelle globale des composés chimiques émis dans la région. Pour Amma, il ne s'agit pas seulement de valider et d'améliorer chacun des modèles composant cette mosaïque, mais aussi et surtout de développer les échanges entre communautés de modélisateurs pour favoriser une compréhension de la MAO dans son ensemble, ce qui permettra de prévoir son évolution dans toutes ses composantes et pour toutes les échelles.

Les modèles de petite échelle qui représentent au plus près les processus de la nature seront confrontés directement aux observations faites sur le terrain et serviront à l'élaboration de leurs représentations plus conceptuelles dans les modèles de grande échelle. Cette méthodologie est une étape indispensable afin de mieux prévoir l'évolution de la mousson des échelles intrasaisonnières à interannuelles et dans le cadre du changement global du climat.

Cet effort d'amélioration des représentations des processus sera aussi effectué dans les modèles de prévision météorologique quotidienne. Pour ces derniers, une meilleure estimation des conditions initiales attendues grâce aux observations Amma contribuera de concert à l'amélioration des prévisions sur cette région. En coopération étroite avec le programme Thorpex, des études d'assimilation des observations et de leurs impacts sur la qualité des prévisions seront réalisées. Des recommandations pourront alors être

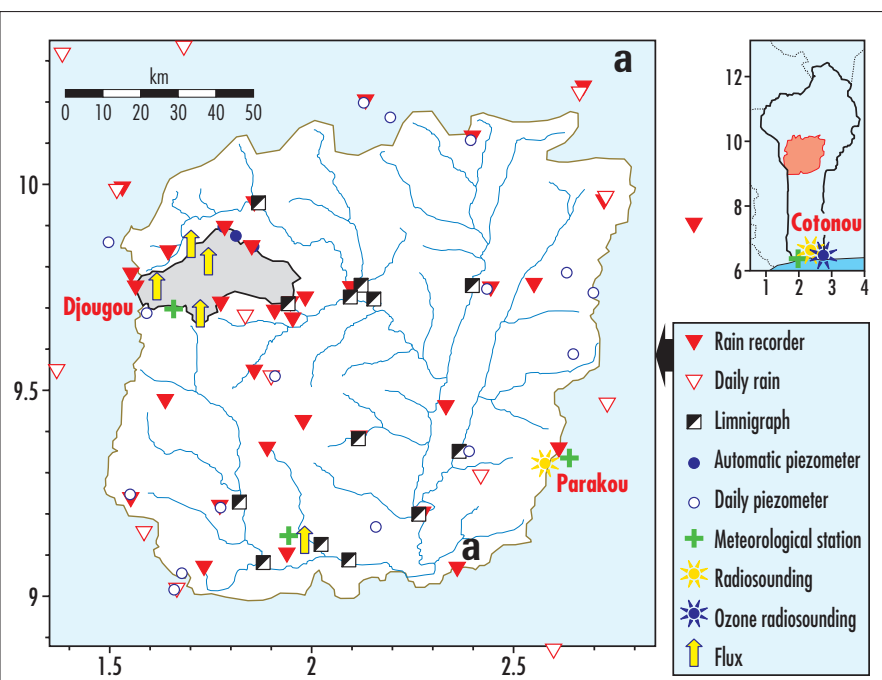

constitue un outil transversal aux diverses thématiques du projet et doit favoriser les collaborations entre les différentes disciplines. Les objectifs généraux sont de donner l'accès le plus simple possible à tous les jeux de données et de leur associer une documentation qui en facilitera l'utilisation. Il s'agit de garantir la pérennité des données pour les études scientifiques au-delà du projet Amma. Un « miroir » de cette base de données sera créé à Niamey pour le bénéfice des scientifiques africains.

\section{Organisation nationale et internationale}

En France, le programme Amma rassemble des chercheurs des disciplines des sciences environnementales et est financé par l'ensemble des agences françaises et l'Union européenne. D'initiative française, il regroupe maintenant des scientifiques de plus de 30 pays et 140 institutions, bénéficiant de l'existence de plusieurs projets Amma fédérateurs dans d'autres pays et de consortium pannationaux : - Amma-Afrique pour les activités des chercheurs des universités africaines

formulées pour un système d'observation opérationnel en Afrique qui permettra de mettre en œuvre les prévisions dont les sociétés africaines ont besoin.

\section{Base de données}

La base de données mise en place dans le cadre du projet Amma a pour but d'archiver de façon pérenne les différents types de données accumulées pendant le projet et de permettre leur exploitation optimale. Elle concerne les observations locales (mesures au sol, aéroportées, en mer...) et satellitaires, ainsi que les résultats de modèles. Elle et de centres opérationnels et de recherche nationaux et régionaux ;

- Amma-EU à travers un projet intégré financé par la Commission européenne ; - Amma-UK et Amma-US pour un ensemble de projets financés respectivement par le Nerc, le DOE, la Nasa et la NOAA.

La coordination internationale est assurée par l'International Scientific Steering Committee (ISSC) et l'International Implementation and Coordination Group (ICIG), sous le contrôle de l'International Governing Board (IGB) qui réunit les principales agences finançant Amma. Un bureau de projet assiste le travail de ces comités. 
Amma est labellisé par le Programme mondial de recherche sur le climat (WCRP) et ses deux composantes Clivar et Gewex, ainsi que par le Programme international géospherebiosphère (IGBP) et ses deux composantes Igac et Ileaps. Amma a aussi des liens de travail avec d'autres projets internationaux dont Gcos, Goos et Thorpex.

\section{Remerciements}

Basé sur une initiative française, Amma a été élaboré par un groupe international de scientifiques et est actuellement financé par un grand nombre d'agences, spécialement en France, au Royaume-Uni, aux ÉtatsUnis d'Amérique et en Afrique. Amma a reçu une contribution majeure de la Communauté européenne dans le cadre du sixième PCRD. Des informations détaillées sur la coordination et les financements peuvent être trouvées sur le site international d'Amma [http:// www.amma-international.org].

Le projet Amma n'aurait pu être lancé sans le soutien des organismes de recherche français et la confiance qu'ils ont placée dans le projet scientifique dès le début. Les programmes de l'Insu (Patom, PNCA, PNEDC, PNTS, PNRH, PNBC) ont permis de construire une base solide à cet édifice complexe, avec les contributions du Cnes, du CNRS, de l'Ifremer, de l'IRD, de Météo-France, des universités françaises et des ministères de la Recherche et des Affaires étrangères. Un projet si ambitieux n'a pas pu se construire sans des efforts coordonnés de très nombreuses personnes, depuis les personnels administratifs jusqu'aux directions des organismes en passant par les personnels de la DT/Insu et de Safire mis à lourde contribution. Nous aimerions toutes les remercier pour leur confiance, leur enthousiasme et leur contribution à Amma.

\section{Exemple de travaux Amma-France relatifs aux impacts santé}

\section{Pour les aspects sur les méningites}

Ont été réalisés en 2005 :

- L'analyse de la variabilité interannuelle de la méningite en Afrique de l'Ouest a commencé au Locean en collaboration avec le LOA et Prodig. Au LOA, l'étude de la relation climat/méningite/aérosol à l'échelle interannuelle sur différents pays de l'Afrique de l'Ouest est en cours.

- L'analyse statistique des données hebdomadaires d'aérosol et de méningites à l'échelle du district au Niger en 2003 (mesures sol et satellitaires d'aérosols, et données météorologiques de température et d'humidité) a été faite au LOA.

- Au Lisa, la modélisation des émissions et concentration en aérosols désertiques en Afrique de l'Ouest a été entreprise ainsi que les simulations préliminaires qui ont été comparées avec les épaisseurs optiques mesurées au sol au Niger, Mali, Sénégal, Cap-Vert en saison sèche.

- Une étude est en cours au Gemi en collaboration avec Prodig et Locean pour mieux comprendre la diffusion et la dynamique des épidémies (à l'échelle interannuelle et interpays africains, d'une part, saisonnière à l'intérieur d'un même pays, d'autre part). Des efforts sont actuellement faits pour identifier les forçages extérieurs sur cette dynamique.

Sont en cours cette année :

- Construction des bases de données communes méningites et aérosols (issus de Toms) et analyse de la variabilité spatiale des dates de démarrage des épidémies au Mali.

- Construction d'indices du contenu en poussières (issus de Toms) et mise en relation avec les épidémies au Mali et sur l'ensemble de l'Afrique de l'Ouest.

\section{Pour les aspects sur le paludisme}

Ont été réalisés en 2006 :

- Une mission de terrain a été effectuée au Sénégal au mois de février à l'IRD Dakar (Laboratoire de paludologie) puis étendue au Niger (IRD, Cermes...). Elle a permis d'obtenir des données terrain décadaires sur 10 ans à l'échelle de villages ou districts. Elles sont de divers types, entomologique, parasitologique, morbidité et mortalité palustres et sont réparties par classe d'âge.

- Ces données font l'objet d'un accord avec leurs auteurs pour leur utilisation au sein d'Amma. Les sites sélectionnés se trouvent au Sénégal : les villages de Ndiop et de Dielmo (taux de piqûres par personne et par nuit, taux d'inoculation entomologique, nombre décadaire d'accès palustres). Les données pluviométriques des postes de Toubacouta, Sokone et Missirah sont associées, ainsi que celles de Kaolack la station synoptique la plus proche des deux villages cités.

- La base de données Mara/Arma a été travaillée au CRC (recherche clinique et épidémiologique) pour croiser données climatiques et épidémiologiques sur la région Amma et montrer les limites de cette base de données. L'étude concerne les variabilités intrasaisonnières et interannuelles des paramètres climatiques et entomologiques et est complétée depuis juin 2005 par un travail sur l'impact des paramètres environnemento-météorologiques sur la densité des vecteurs du paludisme dans cette zone.

- Une coopération avec l'équipe d'A. Morse à Liverpool a été engagée à Locean pour comparer les modèles de dynamique de population d'anophèles (comparaison des sorties de modèle avec des forçages climatiques de différentes régions tropicales).

Sont en cours en 2006 :

- Une étude sur l'impact du climat (cycle moyen annuel, variabilité interannuelle et intrasaisonnière) sur les données issues des analyses parasitologiques (pourcentage de moustiques infectés, etc.), après correction de certaines données terrain et intégration des résultats statistiques dans le modèle de dynamique des populations de moustique anophèles développé à Locean.

- Mission-terrain de 20 jours prévue d'avril à juin sur les sites de Ndiop et Dielmo au Sénégal.

\section{Bibliographie}

\footnotetext{
- Ali A., T. Lebel et A. Amani, 2006: Sahelian rainfall index computation: sensitivity analysis on spatial and time sampling. Journal of International Climatology, soumis.

- Bazzaz F. et W. Sombroek, 1996 : Global climate change and agricultural production. Direct and indirect effects of changing hydrological, pedological and plant physiological processes. John Wiley, FA0, 345 p.

- Craig M.H., R. W. Snow et D. Le Sueur, 1999 : A climate-based distribution model of malaria transmission in sub-Saharan Africa. Parasitology Today, $15,105-111$.

- ISP, 2005 : The International Science Plan for Amma, 103 p. [http://www.amma-international.org/]

Le Barbé L., T. Lebel et D. Tapsoba, 2002 : Rainfall variability in West Africa: a hydrological perspective. J. Climate, 15, $187-202$.

- Redelsperger J.-L., A. Diongue, A. Diedhiou, J.-P. Céron, M. Diop, J.-F. Guérémy, et J.-P. Lafore, 2002 : Multi-scale description of a Sahelian synoptic weather - system representative of the West African monsoon. Quart.J. Roy. Meteoro. Soc., 128, 1229-1257.

- Sultan B. et S. Janicot, 2003 : The West African monsoon dynamics. Part II: The pre-onset and the onset of the summer monsoon. J. Climate, 16, 3407-3427.

- Sultan B., K. Labadi, J.-F. Guégan et S. Janicot, 2005 : Climate drives the meningitis epidemics onset in West Africa. Plos Medicine, Vol. 2, n 1, 43-49.

- UNDP, 2004 : Reducing disaster risk: a challenge for development. UNDP global report, Pelling M. (Ed.), 146 p.
} 